\section{Allgemeinanästhesie}

Anaesthesist 2020 $69: 254-261$ https://doi.org/10.1007/s00101-020-00755-0 Online publiziert: 12. März 2020

(c) Der/die Autor(en) 2020

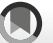

R. Fantin 1 C. M. Ortner ${ }^{2} \cdot$ K. U. Klein ${ }^{3} \cdot$ G. Putz' $\cdot$ D. Marhofer ${ }^{3} \cdot$ S. Jochberger ${ }^{1}$

'Universitätsklinik für Anästhesie und Intensivmedizin, Medizinische Universität Innsbruck, Innsbruck, Österreich

${ }^{2}$ Department of Anesthesiology, Perioperative and Pain Medicine, Stanford School of Medicine, Stanford, USA

${ }^{3}$ Allgemeine Intensivmedizin und Schmerztherapie, Medizinische Universität Wien, Universitätsklinik für Anästhesie, Wien, Österreich

\title{
Spinalanästhesieinduzierte Hypotension bei Sectio caesarea
}

\section{Aktuelle Behandlungskonzepte}

Fundierte Kenntnisse zur Behandlung einer Hypotension nach Spinalanästhesie im Rahmen einer Sectio caesarea sind für jeden Anästhesisten essenziell, um maternale und fetale Komplikationen zu vermeiden. Empfehlungen dazu divergieren allerdings je nach Literatur.

Das im Folgenden von der Arbeitsgruppe geburtshilfliche Anästhesie (ARGE) der Österreichischen Gesellschaft für Anästhesiologie, Reanimation und Intensivmedizin (ÖGARI) kommentierte Versorgungskonzept basiert auf den aus dem Englischen übersetzten und zusammengefassten Empfehlungen von Kinsella et al., welche 2018 publiziert wurden und auf aktuellen internationale Leitlinien beruhen [15].

\section{Definition der Hypotension}

In der Literatur lassen sich unterschiedliche Definitionen einer durch Spinalanästhesie entstehenden Hypotension finden. Je nach Studiendesign wird sich auf absolute systolische Blutdruckwerte (von 80 bis $100 \mathrm{~mm} \mathrm{Hg}$ ), eine von einem Ausgangswert ausgehende prozentuale $\mathrm{Ab}$ nahme bis $30 \%$ oder letztlich eine Kombination davon bezogen. Als Folge dieser

Männliche Funktionsbezeichnungen in diesem Artikel sind geschlechtsneutral zu lesen.
Diskrepanzen ergibt sich eine Inzidenzbreite der Hypotension von 7-74\% [16].

Zwar ist zur Evaluierung einer suffizienten Organperfusion der mittlere arterielle Druck dem systolischen Wert vorzuziehen, allerdings liegen zu dessen Miteinbeziehung in die Definition der Hypotension noch unzureichende Daten vor.

Anmerkung der Arbeitsgruppe geburtshilfliche Anästhesie. Zur Vermeidung falsch-hoher Werte durch Aufregung, Lagerung oder technische Manipulation sollten ggf. Messungen aus dem Mutter-Kind-Pass bzw. der Krankenakte berücksichtigt werden. Auch das Vorliegen einer im Vorfeld diagnostizierten schwangerschaftsinduzierten Hypertonie (SIH) und die Einnahme von antihypertensiver Medikation sind $z u$ evaluieren. Für eine korrekte Messung ist zudem, insbesondere bei der steigenden Zahl adipöser Patientinnen, auf die Verwendung einer passenden Blutdruckmanschette zu achten. Je nach klinischer Ausgangssituation kann mit verstärktem (z.B. vorzeitige Plazentalösung) oder vermindertem ( $z$. B. Präeklampsie) Blutdruckabfall gerechnet werden.

\section{Auswirkungen der Hypotension}

Die durch den Blutdruckabfall induzierte zerebrale Minderperfusion führt zur Aktivierung des Brechzentrums. Basierend auf einer verminderten zerebralen Sauerstoffsättigung kann durch externe Sauerstoffzufuhr eine Besserung der Übelkeit ermöglicht werden. Weiters folgt durch die verringerte Durchblutung des Splanchnikusgebiets die Freisetzung emetogener Faktoren [3, 14].

Bezüglich der fetalen Auswirkungen liegen nur begrenzte Daten vor, allerdings sind durch eine anhaltende uterine Blutflussabnahme Acidämien sowie ein signifikanter Anstieg von Lipidperoxiden im Nabelschnurblut nachgewiesen [25]. Die klinische Relevanz dazu ist jedoch umstritten.

Anmerkung der Arbeitsgruppe geburtshilfliche Anästhesie. Neben der apparativen Überwachung ist die klinische Evaluierung der Patientin essenziell. Die mit einem Blutdruckabfall einhergehenden möglichen Symptome wie Kaltschweißigkeit, Unruhe, Somnolenz, Übelkeit, Dyspnoe oder Parästhesien in den Fingern können pathologischen Messwerten vorausgehen und erfordern eine unverzügliche Therapie. Insbesondere das Risiko einer in diesem Zusammenhang häufig eintretenden perioperativen Übelkeit kann durch eine erfolgreiche Prävention der arteriellen Hypotonie signifikant reduziert werden. Dadurch und durch Vermeidung weiterer Komplikationen kann das subjektive Geburtserlebnis der Mutter hochsignifikant verbessert werden.

\section{Präoperative Vorhersage einer Hypotension}

Anhand von Analysen von Bevölkerungsdaten wurde versucht, individuelle 
Patientenfaktoren als Einflusswert einer Hypotension zu beschreiben. Dabei fand sich ein möglicher Bezug zum BodyMass-Index [20]. Das Heranziehen der präoperativen Herzfrequenz als Vorhersagewert einer Hypotension ist umstritten [2]. Auch ein höherer präoperativer Angstpegel könnte mit einem größeren Abfall des systolischen arteriellen Blutdrucks assoziiert sein [26].

Anmerkung der Arbeitsgruppe geburtshilfliche Anästhesie. Eine nach Erscheinen dieses Konsensus-Statements publizierte Studie zeigte, dass durch einen präoperativ durchgeführten „Passive-legraising"-Test mit $45^{\circ}$ und Evaluation der Abweichungen des Schlagvolumens mittels transthorakaler Echokardiographie eine arterielle Hypotension nach Spinalanästhesieanlage zuverlässig vorhergesagt werden kann. Bei Veränderungen des ,velocity time integral“ $\leq 8 \%$ war das Auftreten einer arteriellen Hypotension als unwahrscheinlich, bei Veränderungen $\geq 21 \%$ als wahrscheinlich einzustufen [29].

Bis eine definitive und breit verfügbare Methode zur Vorhersage vorliegt, wird davon ausgegangen, dass eine rezente Vorgeschichte von spinalanästhesieinduzierten Hypotensionen mit einer höheren Wahrscheinlichkeit zur Entwicklung eines Blutdruckabfalls einhergeht.

Anmerkung der Arbeitsgruppe geburtshilfliche Anästhesie. Aufgrund dessen empfiehlt es sich, frühere Narkoseprotokolle im Hinblick auf den Vasopressorbedarfoder aufgetretene Komplikationen $z u$ überprüfen.

\section{Vasopressorzufuhr bei elektiver Sectio caesarea}

\section{Medikamentenwahl}

Lange Zeit wurde Ephedrin zur Behandlung von Hypotensionen bei einer Spinalanästhesie für eine Sectio caesarea favorisiert. Aufgrund des transplazentaren Übertritts mit Einfluss auf den fetalen Metabolismus gerät die Verwendung von Ephedrin allerdings zunehmend in den Hintergrund [23].
$\mathrm{Zu}$ Prävention und Behandlung von Blutdruckabfällen nach Spinalanästhesie sind Vasopressoren mit vorwiegend a-agonistischer Wirkung zu präferieren. Dabei weist Phenylephrin die besten Ergebnisse auf [11, 12]. Hinsichtlich der Therapie des bradykarden Reflexes und der Herzzeitvolumenabnahme ist aufgrund der zusätzlichen $\beta$-adrenergen Effekte Noradrenalin vorteilhaft [21].

$\mathrm{Zu}$ beachten ist dabei die Vielzahl von Verdünnungsmöglichkeiten. Um das Risiko von Medikationsfehlern zu minimieren, sind klare Vorgehensweisen zu Verdünnungsstandards in den jeweiligen Kliniken obligat.

Anmerkung der Arbeitsgruppe geburtshilfliche Anästhesie. Das Kombinationspräparat Cafedrin-Theodrenalin (Akrinor ${ }^{\circledR}$ ) findet insbesondere in Deutschland häufigen Einsatz und langjährige Anwendungserfahrung. Bisher ließen sich keine maßgeblich negativen Effekte auf die uteroplazentare Versorgung, den kindlichen pH-Wert oder den Apgar-Score nachweisen, wobei eindeutige klinische Daten fehlen [6]. Zu beachten sind die vergleichsweise hohen Kosten sowie die dosisabhängige und verhältnismäßig lange Wirkung. Die Steigerung des Herzzeitvolumens erfolgt primär durch Inotropiezunahme und nur geringfügig durch eine Anhebung des peripheren vaskulären Widerstands. Dies gilt es, in Anbetracht der durch Sympathikolyse bedingten Vasodilatation im Rahmen einer Spinalanästhesie zu bedenken.

Eine kürzlich publizierte retrospektive Analyse mit einer Gegenüberstellung der vasoaktiven Substanzen Ephedrin, Phenylephrin und Akrinor ${ }^{\circledR}$ zeigte nur geringe klinisch relevante Unterschiede im Hinblick auf die Effektivität und fetalen Auswirkungen [4].

Eine rezente Studie von Mohta et al. weist nach Noradrenalingabe einen, im Vergleich zur Phenylephrinapplikation, niedrigeren umbilikalen $\mathrm{pH}$-Wert nach [19]. Dementsprechend ist der Bedarf an weiteren Forschungsbestrebungen gegeben.

Für eine schnelle Applikation empfiehlt es sich, Phenylephrin, Ephedrin sowie Glycopyrronium in Spritzen aufgezogen und mit der genauen Konzentration beschriftet vorliegen zu haben.

\section{Zielblutdruck}

Variierende Definitionen der Hypotension erschweren eine einheitliche Konsensfindung. Kinsella et al. empfehlen, den systolischen arteriellen Druck auf $\geq 90 \%$ des vor Anlage der Spinalanästhesie gemessenen Ausgangswerts bis zur Entbindung zu erhalten. Systolische Blutdruckwerte $<80 \%$ des Ausgangswerts sollten darüber hinaus mit Vasopressorboli behandelt werden.

\section{Blutdruckmessung}

Im klinischen Alltag erfolgt vor Anlage der Spinalanästhesie meist nur eine einzelne Messung zur Ermittlung des Ausgangswertes. Ist dieser bei Frauen ohne vorbekannte Hypertension erhöht, werden wiederholte Messungen nahegelegt.

Anmerkung der Arbeitsgruppe geburtshilfliche Anästhesie. Bis zur Geburt sind engmaschige Blutdruckkontrollen im Abstand von jeweils 1 min empfohlen. Je nach klinischer Stabilität ist anschließend eine Verlängerung der Intervalle möglich. Gegebenenfalls kann zwischen den Intervallen der Blutdruck manuell getastet werden, wobei eine adäquate Beurteilung häufig schwierig ist und gewisse Erfahrung voraussetzt.

\section{Vasopressorapplikation}

Studien zur prophylaktischen Vasopressorbehandlung verglichen die Zufuhr via Infusion oder als Bolusgabe. Als vorteilhaft erwies sich dabei die kontinuierliche Zufuhr von Phenylephrin als Infusion [28]. Zwar geht diese Variante mit einer höheren Gesamtdosis von Phenylephrin einher, jedoch zeigte sich keine Risikozunahme von maternalen Hypertensionen oder Bradykardien [10]. Aufgrund dessen sprechen sich Kinsella et al. für den sofortigen Beginn der kontinuierlichen Vasopressorzufuhr nach Anlage der Spinalanästhesie aus.

Anmerkung der Arbeitsgruppe geburtshilfliche Anästhesie. Im angloamerika- 
nischen Raum stellt die prophylaktische kontinuierliche Infusion von Phenylephrin über einen Infusionsperfusor mit Start unmittelbar bei Gabe des intrathekalen Lokalanästhetikums den „standard of care"dar.

Eine mögliche Variante ist der Start eines Phenylephrinperfusors mit einer Dosierung von $0,5 \mu \mathrm{g} / \mathrm{kgKG}$ und $\mathrm{min}$ bei intrathekaler Gabe des Lokalanästhetikums zur Spinalanästhesie. Bei Bedarf können die Laufrate nach Hämodynamik adaptiert sowie Vasopressorenboli additiv zur kontinuierlichen Therapie appliziert werden (Abb. 1).

Ngan Kee et al. demonstrierten, dass die Verwendung eines Noradrenalinperfusors zur Vermeidung von Hypotension nach Spinalanästhesie als eine sichere Alternative ohne neonatalen Einschränkungen angesehen werden kann [24]. Eine mögliche Variante wäre eine Konzentration von $20 \mu \mathrm{g} / \mathrm{ml}$ und einer initialen Laufrate von $5 \mathrm{ml} / \mathrm{h}$ sowie weiterer Anpassung an die Hämodynamik.

\section{Optimale Vasopressordosis}

Im Hinblick auf die ideale Dosierung von Phenylephrininfusionen erwies sich eine initiale Laufrate von $25-50 \mu \mathrm{g} / \mathrm{min}$ mit nachfolgender individueller Titration als vorteilhaft [1].

Im Vergleich zu einer Bolusgabe des Vasopressors nach spinaler Injektion wird der effektive Blutplasmaspiegel bei kontinuierlicher Infusionsgabe mit einer deutlichen zeitlichen Verzögerung erreicht. Kuhn et al. zeigten, dass ein initialer Phenylephrinbolus von 0,25 $\mu \mathrm{g}$ / kgKG mit einer darauffolgenden Infusion von $0,25 \mu \mathrm{g} / \mathrm{kgKG}$ und min den systolischen Blutdruckwert ohne relevante Nebenwirkungen erhalten konnte [17].

Sollte während der Geburt der Umstieg auf eine Sectio nötig sein, wird der Blutdruckabfall nach Anlage der Spinalanästhesie als deutlich geringer beschrieben, im Vergleich zu elektiven Fällen [22]. Deshalb empfiehlt es sich, die Vasopressorinfusion mit einer niedrigeren Laufrate $\mathrm{zu}$ beginnen.

Anmerkung der Arbeitsgruppe geburtshilfliche Anästhesie. Der meist weniger

Anaesthesist 2020 - 69:254-261 https://doi.org/10.1007/s00101-020-00755-0

(c) Der/die Autor(en) 2020

R. Fantin · C. M. Ortner · K. U. Klein · G. Putz · D. Marhofer · S. Jochberger Spinalanästhesieinduzierte Hypotension bei Sectio caesarea.
Aktuelle Behandlungskonzepte

Zusammenfassung

Eine arterielle Hypotension nach Durchführung einer Spinalanästhesie im Rahmen einer Sectio caesarea kann maternale sowie fetale Nebenwirkungen verursachen. Aktuelle Leitlinien empfehlen eine kontinuierliche und vorzugsweise prophylaktische Anwendung von Vasopressoren, insbesondere a-Agonisten wie Phenylephrin. Neben einer linkslateralen Uterusverschiebung wird ein „intravenous preloading" mit Kolloiden oder ein "coloading" mit Kristalloiden nahegelegt. Zielwert ist, den arteriellen systolischen Blutdruck $\geq 90 \%$ des ermittelten Ausgangswertes zu halten bzw. einen Abfall auf $<80 \%$ des Ausgangswertes zu verhindern.
Dafür wird eine prophylaktische Zufuhr von Phenylephrin via Perfusor mit verstellbarer Laufrate empfohlen. Diese sollte mit einer Dosierung von $25-50 \mu \mathrm{g} / \mathrm{min}$ nach intrathekaler Injektion des Lokalanästhetikums unverzüglich gestartet und nach Vitalwerten titriert werden. Schwangere mit kardialen Vorerkrankungen gilt es, präoperativ individuell zu evaluieren.

Schlüsselwörter

Geburtshilfliche Anästhesie · Blutdruck · Hämodynamische Instabilität · Vasopressor . Phenylephrin

\section{Hypotension induced by spinal anesthesia during cesarean section. Current treatment concepts}

\section{Abstract}

Arterial hypotension during cesarean delivery under spinal anesthesia can cause maternal and fetal adverse effects. Therefore, current guidelines recommend the continuous and preferably prophylactic use of vasopressors, emphasizing the use of alpha-agonists, such as phenylephrine. Besides a left lateral uterine displacement either an intravenous colloid preloading or a crystalloid co-loading is recommended. The blood pressure goal is to maintain a systolic arterial blood pressure of at least $90 \%$ of the initial baseline value and to avoid a drop to less than $80 \%$ of this baseline. To achieve this goal a prophylactic continuous phenylephrine infusion with an adjustable flow rate is recommended. It is advised to start with an initial dose of $25-50 \mu \mathrm{g} / \mathrm{min}$, initiated immediately following the intrathecal injection of the local anesthetic and titrated according to the vital parameters. Parturients with cardiac diseases should be preoperatively evaluated following individual hemodynamic goals.

Keywords

Obstetric Anaesthesia - Blood Pressure . Hemodynamic Instability · Vasopressor . Phenylephrine ausgeprägte Blutdruckabfall nach Anlage der Spinalanästhesie bei Umstieg auf eine Sectio basiert auf der bestehenden Wehentätigkeit. Durch die regelmäßigen Uteruskontraktionen werden somit größere Blutmengen in den mütterlichen Kreislauf befördert.

\section{Medikamente der zweiten Wahl}

Nach Verwendung eines a-Agonisten ist bei niedriger Herzfrequenz und einem systolischen Blutdruckwert $<90 \%$ des Ausgangswertes die Applikation von Ephedrin zu befürworten. Hinsichtlich einer Bradykardie ohne gleichzeitig vor- liegende Hypotension liegt kein unterer Grenzwert zur Initiierung einer Therapie vor. Im Fall einer Bradykardie mit Hypotension bietet sich die Gabe eines Anticholinergikums (Glycopyrronium oder Atropin) an. Für eine routinemäßige Gabe des Anticholinergikums in präventiver Absicht mangelt es allerdings an ausreichenden Belegen [27].

\section{Anmerkung der Arbeitsgruppe geburts-} hilfliche Anästhesie. Da Atropin, im Gegensatz zur Glycopyrronium, die Plazentaschranke überwinden und damit die fetale Herzfrequenz beeinflussen kann, wird primär die Gabe von Glycopyrronium bei 
Hier steht eine Anzeige.

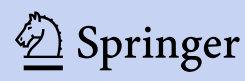




\section{Allgemeinanästhesie}

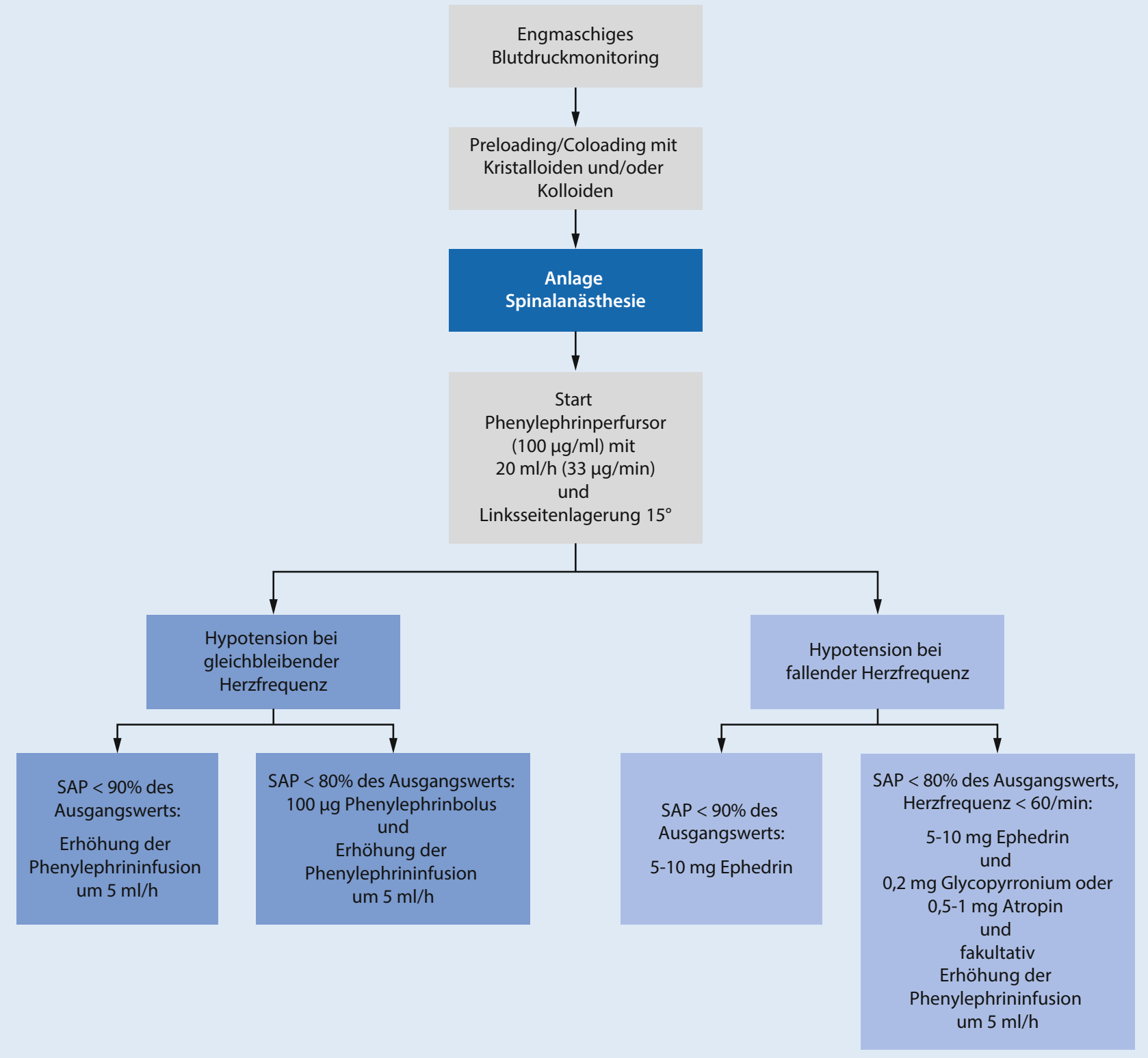

Abb. 1 ॥ Empfehlung für manuell titrierte Vasopressorinfusionen bei elektiver Sectio caesarea in Spinalanästhesie. SAPSystolic arterial pressure (systolischer arterieller Druck). Modifiziert nach Kinsella [15]

therapiebedürftigen Bradykardien empfohlen. Bei Auftreten von Bradykardien bzw. Tachykardien sollten alternative Ursachen, wie $z$. B. eine aufsteigende spinale Blockade, Blutungen oder auch medikamentöse Nebenwirkungen, in Erwägung gezogen werden.

\section{Weitere Behandlungs- möglichkeiten}

Zusätzliche Optionen zu Vermeidung und Therapie hämodynamischer Insta- bilität beinhalten Methoden zur Minderung der Kompression der V. cava inferior und des venösen Pooling in die unteren Extremitäten [16]. Dabei wird eine Linksseitenlagerung von $15^{\circ}$ durch Kippen des OP-Tisches oder eine Keilunterlage empfohlen.

Studien zur Verwendung von venösen Kompressionsverfahren beschreiben unterschiedliche Ergebnisse, da der Vorteil von der Art und Intensität der verwendeten Kompressionsmethoden abhängt [7]. Basierend auf dem geringeren Ein- fluss der Venodilatation nach Spinalanästhesie, verglichen mit der arteriellen dilatativen Komponente, ist die venöse Kompression von geringfügigem Nutzen.

Anmerkung der Arbeitsgruppe geburtshilfliche Anästhesie. Im Hinblick auf die Effizienz von Lagerungstechniken finden sich in der Literatur kontroverse Meinungen.

Radiologisch zeigt sich eine sichtbare Entlastung der $V$. cava inferior erst ab einem Neigungswinkel von $30^{\circ}$ [13]. Ei- 
ne Lagerung in diesem Ausmaß kann, neben erschwerten Operationsbedingungen, auch zum Unwohlsein der Mutter führen. Die ARGE empfiehlt, gemäß internationaler Guidelines, grundsätzlich eine Linksseitenlagerung von $15^{\circ}$ anzustreben, jedoch stets in Übereinkunft mit dem subjektiven Befinden der Patientin.

Seit einigen Jahren wird die Wirksamkeit des „preloading“ zur Reduzierung der Inzidenz oder des Schweregrads der Hypotension infrage gestellt und vielseitig diskutiert.

Dyer et al. beschrieben einen Vorteil des „coloading“ mit Kristalloiden zum Ausgleich einer Hypotension bzw. zur Senkung des Vasopressorbedarfs [10]. Hinsichtlich der Wahl der verwendeten Flüssigkeit zeigte sich ein Preloading mit Kolloiden effektiver als mit Kristalloiden [7].

\section{Anmerkung der Arbeitsgruppe geburts- hilfliche Anästhesie. Vor Durchführung der Spinalanästhesie sind ein funktio- nierendes Monitoring sowie mindestens ein sicher sitzender und gut zugänglicher Somnolenz unter Reduktion der konti- nuierlichen Vasopressorzufuhr bzw. ein weiterhin bestehender oder ansteigender Vasopressorbedarf können Hinweise für eine latente Blutung sein.} peripherer venöser Zugang zu gewährleisten.

Bei einem erwartet höheren Blutverlust werden für eine möglichst zügige Volumengabe mehrere großlumige venöse Zugänge (16-20G) empfohlen. Sofern möglich, sollte die Blutdruckmanschette am kontralateralen Arm angelegt werden, sodass durch häufige Messungen der Fluss der Infusion nicht reduziert wird. Um bei Bedarf die Flüssigkeitsrate zügig $z u$ erhöhen, kann die Infusion in einem Druckbeutel platziert werden. Nach spinaler Injektion des Lokalanästhetikums sollte eine zügige sowie je nach Barizität passende Lagerung der Patientin erfolgen.

Bezüglich der optimalen Flüssigkeitszufuhr zu Preloading und Coloading besteht weiterhin keine Übereinkunft. Kinsella et al. geben keine eindeutige Empfehlung zu Menge und Art der Volumina an. Ein Preloading darf v.a. bei dringlicher Indikation die Anlage der Spinalanästhesie nicht verzögern. Die DGAI rät in den derzeit geltenden S3-Leitlinien „Intravasale Volumentherapie beim Erwachsenen" (AWMF-Register-Nr. 001/020, Stand 31.06.2014) dezidiert von der standardmäßigen Verwendung von Kolloiden bei
Schwangeren ab. Angesichts der derzeit noch kontroversen Daten zu Effizienz und möglichen Nebenwirkungen empfiehlt die ARGE, den Einsatz kolloidaler Infusionslösungen primär auf Notfallsituationen zu beschränken.

Nach spinaler Injektion des Lokalanästhetikums sollte eine zügige sowie je nach Barizität passende Lagerung der Patientin erfolgen.

\section{Nach der Geburt}

Nach Abnabelung kann ein schrittweises Ausschleichen der kontinuierlichen Vasopressorinfusion unter Beachtung der möglichen hypotensiven Wirkung des während der Geburt verabreichten Oxytozins initiiert werden. Sofern stabile Verhältnisse ohne klinische Symptomatik vorliegen, ist eine relative Hypotension tolerierbar. Übelkeit oder zunehmende

\section{Anmerkung der Arbeitsgruppe geburts-} hilfliche Anästhesie. Das nach Abnabelung applizierte Oxytozin reduziert signifikant den systemisch vaskulären Widerstand und sollte deshalb nicht als Bolus verabreicht werden. Stattdessen empfiehlt sich die Zufuhr als Kurzinfusion über 5 min. Ein Bolus mit höherer Dosis kann insbesondere bei bestehender Hypovolämie durch Blutung bzw. kardialer Vorerkrankung zu einer lebensgefährlichen kardialen Dekompensation führen.

Sofern nach der Geburt ein bestehender oder ansteigender Vasopressorbedarf vorliegt, ist eine frühzeitige Kommunikation mit den Operateuren im Hinblick auf eine Blutung essenziell. Differenzialdiagnostisch gilt es, weitere Pathologien, wie eine unerkannte vorbestehende Herzerkrankung oder eine peripartale Kardiomyopathie, zu erwägen.

\section{Präeklampsie}

Die Hypotensionsrate sowie der Vasopressorbedarf nach Spinalanästhesieanlage sind bei Patientinnen mit Präeklampsie geringer als bei gesunden Frau- en [5]. Möglicherweise lässt sich dies auf eine größere Menge endogener vasoaktiver Mediatoren oder erhöhte Sensitivität auf exogene Vasopressoren zurückführen. Aufgrund des erniedrigten Vasopressorbedarfs ist meist keine initiale Vasopressorinfusion erforderlich bzw. eine geringere Laufrate $\mathrm{zu}$ Beginn ausreichend.

Anmerkung der Arbeitsgruppe geburtshilfliche Anästhesie. Bei beginnender Präeklampsie empfehlen sich eine frühzeitige anästhesiologische Evaluierung und Aufklärung der Patientin für eine geplante oder notfallmäßige Sectio caesarea.

Dabei betonten Dyer et al., dass Phenylephrin bei Präeklampsie und erhaltener systolischer Funktion den Vasopressor der Wahl darstellt [8]. Der neonatale Säure-Basen-Haushalt ist unabhängig von der Gabe von Ephedrin oder Phenylephrin [9]. Die Wahl des Vasopressors kann daher nach dem Hämodynamikstatus gewählt werden. Im Vordergrund stehen die Dynamik des Blutdrucks, der Gerinnung und Blutbildveränderungen im Verlauf.

\section{Kardiale Vorerkrankungen}

Neuroaxiale Techniken gewinnen bei Patientinnen mit kardialen Vorerkrankungen zunehmend an Relevanz. Allerdings werden hämodynamische Veränderungen oft schlecht toleriert, insbesondere bei einer vorlastabhängigen Pathophysiologie oder erforderlichen konstanten Herzzeitvolumina. Gut beschrieben sind titrierbare, katheterbasierte neuroaxiale Techniken wie die Epiduralanalgesie, niedrigdosierte kombinierte Spinal- und Epidualanästhesie oder eine kontinuierliche Spinalanästhesie [18].

Die Wahl des Vasopressors sollte dabei stets von den hämodynamischen Erfordernissen der kardialen Vorerkrankungen beeinflusst werden.

\section{Anmerkung der Arbeitsgruppe geburts-} hilfliche Anästhesie. Je nach Ausprägung der kardialen Pathologie ist eine präoperative Vorstellung in der Anästhesieambulanz mit rezenten kardiologischen Befunden zur fokussierten Anamnese mit Untersuchung und klinischen Beurteilung ratsam. Dabei ist eine ausführliche Auf- 
klärung der Patientin über das erhöhte kardiale Risiko und evtl. weiterer Maßnahmen, wie ein invasives Blutdruckmonitoring, von Bedeutung.

Anstelle einer Spinalanästhesie empfiehlt sich die Anlage einer kombinierten Spinal- und Epiduralanästhesie mit einer langsamen Titration des niedrig dosierten Lokalanästhetikums. Gegebenenfalls ist eine verlängerte postoperative Observation im Aufwachraum oder auf einer Intensivstation erforderlich.

\section{Fazit für die Praxis}

- Trotz zahlreicher Studien fehlen zu einigen Therapiemöglichkeiten weiterhin klare Behandlungsalgorithmen.

- Jede Vermeidung einer Hypotension nach Anlage einer Spinalanästhesie reduziert das Risiko von Übelkeit und Erbrechen der Mutter signifikant und ist somit für das Wohlbefinden bei der Geburt und für die Geburt als ein schönes Erlebnis relevant.

- Neben Lagerungstechniken und adäquater Flüssigkeitszufuhr wird in aktuellen internationalen Leitlinien die prophylaktische kontinuierliche Zufuhr von Vasopressoren, vornehmlich Phenylephrin, empfohlen.

- Das Ziel ist es, den systolischen arteriellen Blutdruck $\geq 90 \%$ der ermittelten Ausgangswerte zu erhalten bzw. einen Abfall $<80 \%$ dieser Baseline zu verhindern.

- Für Patientinnen mit kardialen Vorerkrankungen ist eine perioperative individuelle Evaluation und Behandlung erforderlich.

\section{Korrespondenzadresse}

\section{R. Fantin}

Universitätsklinik für Anästhesie und Intensivmedizin, Medizinische Universität Innsbruck

Anichstraße 35, 6020 Innsbruck, Österreich raffaella.fantin@i-med.ac.at

Funding. Open access funding provided by University of Innsbruck and Medical University of Innsbruck.

\section{Einhaltung ethischer Richtlinien}

Interessenkonflikt. R. Fantin, C. M. Ortner, K. U. Klein, G. Putz, D. Marhofer und S. Jochberger geben an, dass kein Interessenkonflikt besteht.

Für diesen Beitrag wurden von den Autoren keine Studien an Menschen oder Tieren durchgeführt. Für die aufgeführten Studien gelten die jeweils dort angegebenen ethischen Richtlinien.

Open Access Dieser Artikel wird unter der Creative Commons Namensnennung 4.0 International Lizenz veröffentlicht, welche die Nutzung, Vervielfältigung, Bearbeitung, Verbreitung und Wiedergabe in jeglichem Medium und Format erlaubt, sofern Sie den/die ursprünglichen Autor(en) und die Quelle ordnungsgemäßnennen, einen Link zur Creative Commons Lizenz beifügen und angeben, ob Änderungen vorgenommen wurden.

Die in diesem Artikel enthaltenen Bilder und sonstiges Drittmaterial unterliegen ebenfalls der genannten Creative Commons Lizenz, sofern sich aus der Abbildungslegende nichts anderes ergibt. Sofern das betreffende Material nicht unter der genannten Creative Commons Lizenz steht und die betreffende Handlung nicht nach gesetzlichen Vorschriften erlaubt ist, ist für die oben aufgeführten Weiterverwendungen des Materials die Einwilligung des jeweiligen Rechteinhabers einzuholen.

Weitere Details zur Lizenz entnehmen Sie bitte der Lizenzinformation auf http://creativecommons.org/ licenses/by/4.0/deed.de.

\section{Literatur}

1. Allen TK, George RB, White WD et al (2010) A double-blind, placebo-controlled trial of four fixed rate infusion regimes of phenylephrine for hemodynamic support during spinal anesthesia for cesarean delivery. Anesth Analg 111:1221-1229

2. Bishop DG, Cairns C, Grobbelaar M et al (2017) Heart rate variability as a predictor of hypotension following spinal for elective caesarean section: a prospective observational study. Anaesthesia 72(5):603-608

3. Borgeat A, Ekatodramis G, Schenker CA (2003) Postoperative nausea and vomiting in regional anesthesia: a review. Anesthesiology 98:530-547

4. Chapell D, Helf A, Gayer J et al (2019) Antihypotensiva bei Kaiserschnittentbindungen: Behandlung der arteriellen Hypotension mit Ephedrin, Phenylephrin und Akrinor ${ }^{\circledR}$ (Cafedrin/Theodrenalin) im Rahmen von Kaiserschnittentbindungen in Spinalanästhesie. Anaesthesist 68:228-238

5. Clark VA, Sharwood-Smith GH, Stewart AV (2005) Ephedrine requirements are reduced during spinal anaesthesia for caesarean section in preeclampsia. Int JObstet Anesth 14(1):9-13

6. Clemens KE, Quednau I, Heller AR, KlaschikE (2019) Impact of cafedrine/theodrenaline (Akrinor ${ }^{\circledR}$ ) on therapy of maternal hypotension during spinal anesthesia for cesarean delivery: a retrospective study. Minerva Ginecol62(5):514-524

7. Cyna AM, Andrew M, Emmett RS et al (2006) Techniques for preventing hypotension during spinal anaesthesia for caesarean section. Cochrane Database Syst Rev 18(4):CD2251
8. Dyer RA, Daniels A, Vorster A et al (2017) Maternal cardiac output response to colloid preload and vasopressor therapy during spinal anaesthesia for caesarean section in patients with severe pre-eclampsia: a randomized, controlled trial. Anaesthesia 73(1):23-31

9. Dyer RA, Emmanuel A, Adams SC et al (2018) A randomised comparison of bolus phenylephrine and ephedrine for the management of spinal hypotension in patients with severe preeclampsia and fetal compromise. Int J Obstet Anesth 33:23-31

10. Dyer RA, Farina Z, Joubert IA et al (2004) Crystalloid preload versus rapid crystalloid administration after induction of spinal anaesthesia (coload) for elective caesarean section. Anaesth Intensive Care 32(3):351-357

11. Heesen M, Klöhr S, Roissaint R, Straube S (2014) Prophylactic phenylephrine for caesarean section under spinal anaesthesia: systematic review and meta-analysis. Anaesthesia 69(2):143-165

12. Heesen M, Stewart A, Fernando R (2015) Vasopressors for the treatment of maternal hypotension following spinal anaesthesia for elective caesarean section: past, present and future. Anaesthesia 70(3):252-257

13. Higuchi H, Takagi S, Zhang K et al (2015) Effect of lateral tilt angle on the volume of the abdominal aorta and inferior vena cava in pregnant and nonpregnant women determined by magnetic resonance imaging. Anesthesiology 122(2):286-293

14. Hirose N, Kondo Y, Maeda T et al (2016) Oxygen supplementation is effective in attenuating maternal cerebral blood deoxygenation after spinal anesthesia for cesarean section. Adv Exp Med Biol 876:471-477

15. Kinsella SM, Carvalho B, Dyer RA et al (2018) International consensus statement on the management of hypotension with vasopressors during caesarean section under spinal anaesthesia. Anaesthesia 73(1):71-92

16. Klöhr S, Roth R, Hofmann T et al (2010) Definitions of hypotension after spinal anaesthesia for caesarean section: literature search and application to parturients. Acta Anaesthesiol Scand 54(8):909-921

17. Kuhn JC, Hauge TH, Rosseland LA et al (2016) Hemodynamics of phenylephrine infusion versus lower extremity compression during spinal anesthesia for cesarean delivery: a randomized, double-blind, placebo-controlled study. Anesth Analg 122(4):1120-1129

18. Langesaeter E, Dragsund M, Rosseland LA (2010) Regional anaesthesia for a caesarean section in women with cardiac disease: a prospective study. Acta Anaesthesiol Scand 54(1):46-54

19. Mohta M, Garg A, Chilkoti GT, Malhotra RK (2019) A randomised controlled trial of phenylephrine and noradrenaline boluses for treatment of post spinal hypotension during elective caesarean section. Anaesthesia 74(7):850-855

20. Nani FS, Torres ML (2011) Correlation between the body mass index (BMI) of pregnant women and the development of hypotension after spinal anesthesia for cesarean section. Rev Bras Anestesiol61(1):21-30

21. Ngan Kee WD (2017) Norepinephrine for maintaining blood pressure during spinal anaesthesia for caesarean section: a 12-month review of individual use. Int JObstet Anesth 30:73-74

22. Ngan Kee WD, Khaw KS, Lau TK et al (2008) Randomised double-blinded comparison of phenylephrine vs. ephedrine for maintaining 
blood pressure during spinal anaesthesia for non-elective caesarean section. Anaesthesia 63(12):1319-1326

23. Ngan Kee WD, Khaw KS, Tan PE et al (2009) Placental transfer and fetal metabolic effects of phenylephrine and ephedrine during spinal anesthesia for cesarean delivery. Anesthesiology 111(3):506-512

24. Ngan Kee WD, Lee SWY et al (2018) Prophylactic norepinephrine infusion for preventing hypotension during spinal anesthesia for cesarean delivery. Anesth Analg 126(6):1989-1894

25. Okudaira S, Suzuki S (2005) Influence of spinal hypotension on fetal oxidative status during elective cesarean section in uncomplicated pregnancies. Arch Gynecol Obstet 271 (4):292-295

26. Orbach-Zinger S, Ginosar Y, Elliston J et al (2012) Influence of preoperative anxiety on hypotension after spinal anaesthesia in women undergoing caesarean delivery. Br $J$ Anaesth 109(6):943-949

27. Patel SD, Habib AS, Phillips $S$ et al (2018) The effect of glycopyrrolate on the incidence of hypotension and vasopressor requirement during spinal anesthesia for cesarean delivery: a metaanalysis. Anesth Analg 126(2):552-558

28. Sen I, Hirachan R, Bhardwaj N et al (2013) Colloid cohydration and variable rate phenylephrine infusion effectively prevents postspinal hypotension in elective cesarean deliveries. J Anaesthesiol Clin Pharmacol 29(3):348-355

29. Zieleskiewicz L, Noel A, Duclos G et al (2018) Can point-of-care ultrasound predict spinal hypotension during caesarean section? A prospective observational study. Anaesthesia 73(1):15-22

\section{Hier steht eine Anzeige.}

\section{Springer}

